\title{
Eficacia de la Analgesia Preoperatoria con Antiinflamatorios no Esteroidales (Aines) en Cirugía de Terceros Molares. Revisión de la Literatura
}

\author{
Efficacy of Preemptive Analgesia with Nonsteroidal Anti-Inflammatory \\ Drugs (Nsaids) in Third Molar Surgery. Literature Review \\ Ulises Carballosa F. ${ }^{1}$; Gerardo Aguilar Z. ${ }^{2}$; Cristobal Pacheco M. ${ }^{1}$ \& Liberto Figueroa C. ${ }^{3}$
}

CARBALLOSA, F. U.; AGUILAR, Z. G.; PACHECO, M. C. \& FIGUEROA, C. L. Eficacia de la analgesia preoperatoria con antiinflamatorios no esteroidales (Aines) en cirugía de terceros molares. Revisión de la literatura. Int. J. Odontostomat., 12(2):131-136, 2018.

RESUMEN: El manejo del dolor postoperatorio en cirugía de desinclusión de terceros molares es fundamental. El uso de antiinflamatorios no esteroidales (AINES), son considerados como una excelente alternativa para el manejo del dolor, siendo administrados tanto postoperatorios, como preoperatorios. El propósito de la presente revisión bibliográfica fue establecer la eficacia y seguridad de la analgesia preoperatoria con antiinflamatorios no esteroidales en desinclusiones quirúrgicas de terceros molares en las que hubiera remoción parcial o total de hueso. Se realizó una búsqueda en las bases de datos: PubMed, EBSCO, Cochrane Library y Scielo; usando las palabras claves: Preemptive Analgesia, NSAID, Oral Surgery. Trece artículos fueron seleccionados, sumando un total de 875 pacientes evaluados. Si bien varios autores consideraron la analgesia preoperatoria con AINES, como un método eficaz, hacen falta más estudios que demuestren su eficacia en cirugía oral.

PALABRAS CLAVE: analgesia preventiva, AINES, cirugía oral.

\section{INTRODUCCIÓN}

La cirugía oral y maxilofacial genera dolor y edema postoperatorio, debido a su acción sobre tejidos duros y blandos, por lo que el manejo del dolor en este tipo de cirugía es fundamental. El control de estos signos y síntomas ha sido largamente estudiado, determinándose actualmente que su control adecuado es posible, para lo cual el cirujano maxilofacial cuenta con un número bastante amplio de antiinflamatorios no esteroidales con buenas propiedades analgésicas y antiinflamatorias. Los casos de dolores refractarios casi siempre corresponden a factores etiológicos consecutivos a la cirugía, tales como: fracturas, alveolitis, infecciones, etc.

Los esquemas para tratar el dolor son en su mayoría son empíricos y se inician una vez terminado el acto quirúrgico o durante el mismo. Pero existe discrepancia respecto a si la oportunidad de la adminis- tración, pre o postoperatoria modifica el curso del dolor postquirúrgico (Romero-Ruiz et al., 2006).

Ya en los principios del siglo pasado, Katz (1993) observaron que si la transmisión del dolor se bloqueaba antes de la incisión quirúrgica la mortalidad disminuía. Años después, los defensores de esta técnica la llamaron analgesia preoperatoria, y también notaron una disminución en la intensidad y duración del dolor postoperatorio (Kelly et al., 2001a,b).

En los últimos años ha surgido una corriente desde el área de la medicina y la anestesiología que postula la administración preoperatoria de analgésicos como una nueva herramienta para el manejo del dolor postoperatorio, incluso algunos autores concluyen que su administración preoperatoria tendría mejores resultados que su administración postoperatoria (Campbell, 1990).

\footnotetext{
${ }^{1}$ Residente, Cirugía y Traumatología Maxilofacial, Universidad de los Andes, Santiago, Chile.

${ }^{2}$ Cirujano Dentista, Universidad de los Andes, Santiago, Chile.

${ }^{3}$ Cirujano Maxilofacial, Clínica Alemana de Santiago, Santiago, Chile
} 
El concepto de analgesia preoperatoria, para reducir la intensidad y duración del dolor postoperatorio, fue introducido por Woolf en el año 1983, quien mostró evidencia de un componente de hipersensibilidad dolorosa en el SNC, tras una lesión en estudios experimentales (Woolf \& Chong, 1993). También puede ser definida como un tratamiento antinociceptivo que impide el establecimientoalterado en el SNC de los impulsos aferentes, el cual amplifica el dolor postoperatorio (Kissin, 2000).

La analgesia preoperatoria según Grape \& Tramèr (2007), debe tener tres objetivos: el primero de ellos, disminuir el dolor agudo después de la injuria tisular, en la etapa intraoperatoria y postoperatoria; segundo, prevenir la modulación del dolor a nivel del SNC (memoria del dolor); y, por último, inhibir la persistencia del dolor postoperatorio y el desarrollo del dolor crónico.

La analgesia preoperatoria se define entonces, como la administración de un fármaco analgésico antes de que la noxa tisular quirúrgica sea iniciada, bloqueando así la nocicepción periférica y central, logrando como objetivos: disminuir el dolor agudo después de la injuria tisular, prevenir la modulación del dolor a nivel del SNC, y por último inhibir la persistencia del dolor postoperatorio y desarrollo del dolor crónico (Grape \& Tramèr).

Existen diferentes modalidades de analgesia preoperatoria, destacándose el uso de opiodes, anestesia regional, antagonistas de los receptores glutamatérgicos NMDA, y el uso de antiinflamatorios no esteroidales (AINEs) (Kelly et al.2001a,b).

El propósito de esta revisión es establecer si existe eficacia en la administración preoperatoria de AINEs en cirugía de desinclusión de terceros molares.

\section{MATERIAL Y MÉTODO}

Se realizó una revisión en las bases de datos PubMed, EBSCO, Cochrane Library y Scielo, de artículos en inglés y español. Sin límite de año, ocupando las siguientes palabras clave: preemptive analgesia, oral surgery y NSAIDs, en combinación con el término booleano AND.

Del total de los artículos encontrados, se analizaron aquellos que cumplían con los siguientes criterios de inclusión:
1) Ensayos clínicos randomizados

2) Presencia de tercer molar superior o inferior que requiriera remoción parcial o total de hueso.

3) Uso de un AINE administrado en la etapa preoperatoria en algún grupo de estudio

4) Utilización de anestesia local en todas las cirugías orales

Todas aquellas publicaciones basadas en datos en animales, y que ocupasen fármacos preoperatorios como opioides, ansiolíticos, hipnóticos o anestésicos generales, fueron excluidas. También aquellos artículos que tuvieran menos de 10 pacientes por grupo de estudio.

Todos los artículos evaluaron la sensibilidad dolorosa ocupando una escala visual análoga (EVA).

Una vez recolectados los datos, fueron analizados de forma descriptiva en base a variables como: tipo de cirugía, analgésico utilizado, dosis, vía de administración, y si los requerimientos de analgésicos de rescate fueron mayores en el grupo preoperatorio que en los grupos control.

\section{RESULTADOS}

Un total de 154 estudios fueron encontrados en las bases de datos PubMed, EBSCO, Cochrane y Scielo. Del total de artículos, se excluyeron 132 por no cumplir los criterios de inclusión establecidos.

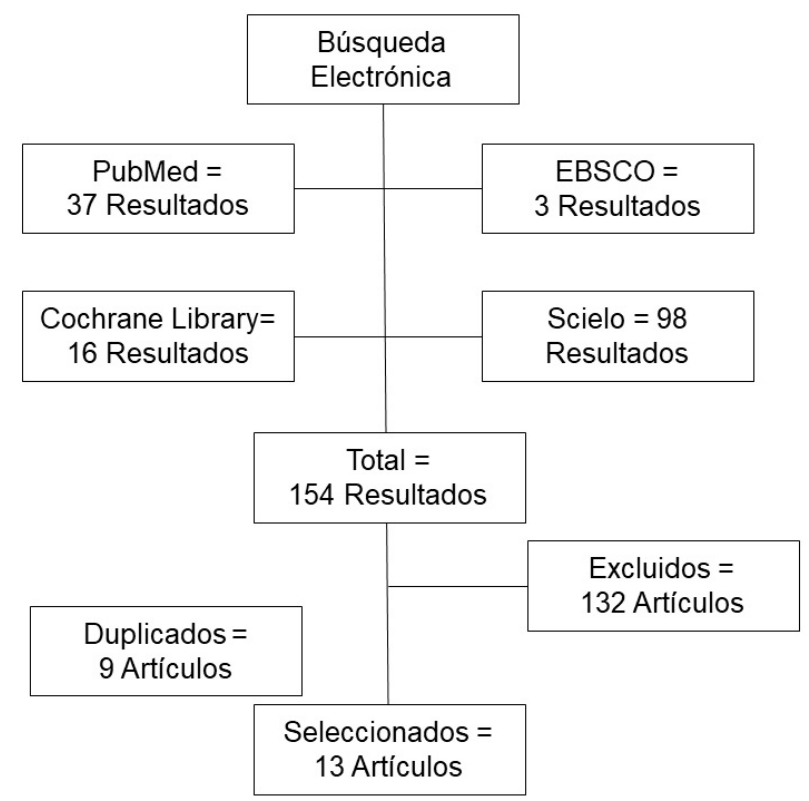

Fig. 1. Flujograma resumen búsqueda. 


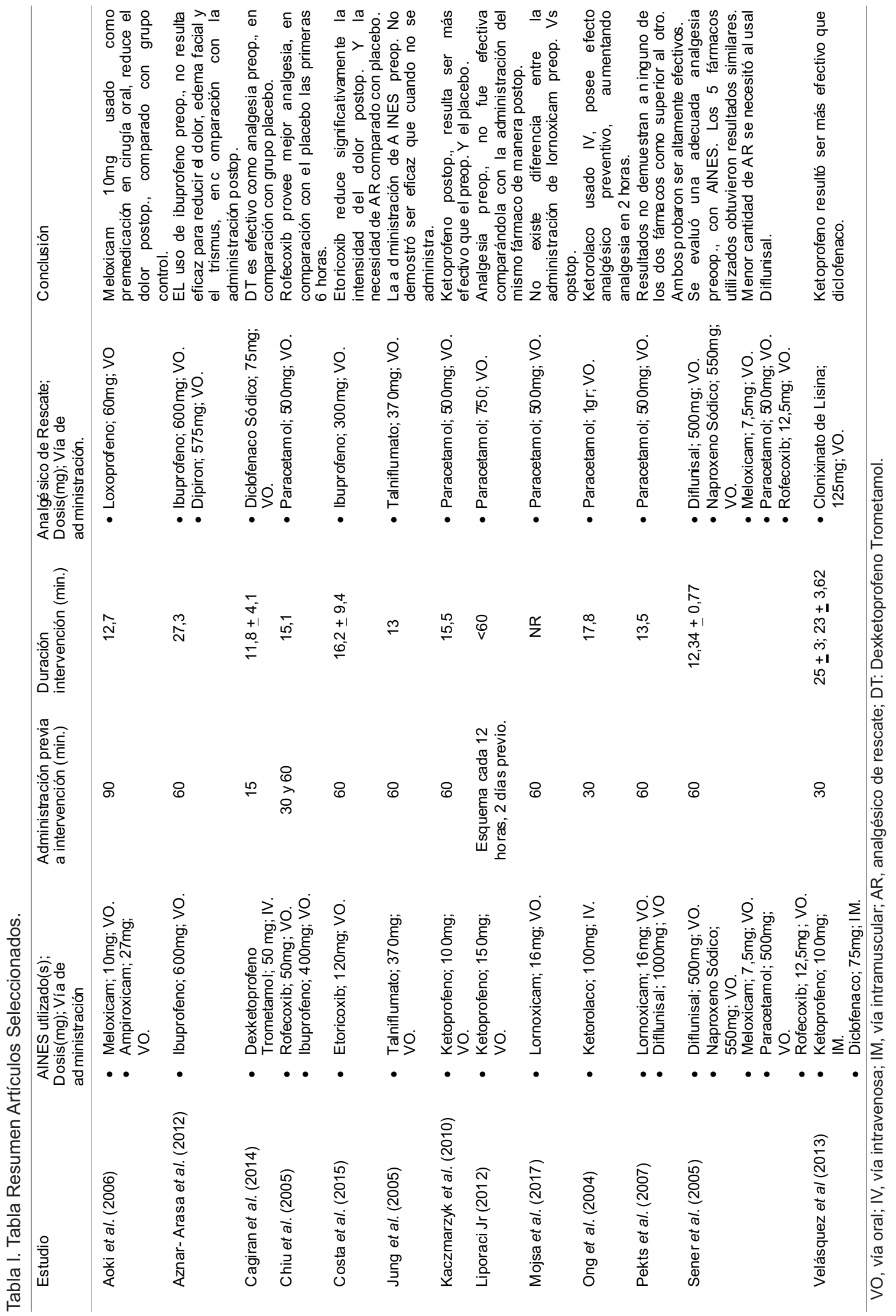


Tabla II. Datos demográficos.

\begin{tabular}{lccc}
\hline Estudio & Número de Pacientes (H:M) & $\begin{array}{c}\text { Promedio Edad } \\
\text { (años) }\end{array}$ & Peso (Kg) \\
\hline Aoki et al. (2006) & $91(34: 57)$ & 25,0 & $\mathrm{NR}$ \\
Aznar- Arasa et al. (2012) & $109(51: 58)$ & 26,2 & $\mathrm{NR}$ \\
Cagiran et al. (2014) & $20(\mathrm{NR})$ & 22,8 & $\mathrm{NR}$ \\
Chiu et al. (2005) & $98(38: 60)$ & 22,5 & $\mathrm{NR}$ \\
Costa et al. (2015) & $18(8: 10)$ & $\mathrm{NR}$ & $\mathrm{NR}$ \\
Jung et al. (2005) & $80(37: 43)$ & 23,9 & $\mathrm{NR}$ \\
Kaczmarzyk et al. (2010) & $96(28: 68)$ & 22,4 & $\mathrm{NR}$ \\
Liporaci Jr (2012) & $13(2: 11)$ & 21 & $\mathrm{NR}$ \\
Mojsa et al. (2017) & $90(31: 59)$ & 24,5 & $\mathrm{NR}$ \\
Ong et al. (2004) & $30(13: 17)$ & 27,8 & $65,2+10,8$ \\
Pekts et al. (2007) & $40(15: 25)$ & 23,7 & $65,2 \pm 2$ \\
Sener et al. (2005) & $150(42: 108)$ & 26,8 & $\mathrm{NR}$ \\
Velásquez et al (2013) & $20(9: 11) ; 20(7: 13)$ & 23,$6 ; 23,9$ & $60,8+9,72 ; 58,41+7,98$
\end{tabular}

NR, No Reportado

Otros 9 artículos se encontraban duplicados, dejando un total de 13 artículos para nuestro análisis, todos en relación con cirugía de desinclusión de terceros molares. Resultados de la búsqueda se detallan en Fig. 1.

Los 13 trabajos se reúne la información de 875 pacientes estudiados. A continuación, se presenta en la Tabla I, el resumen las características demográficas de los pacientes seleccionados en los 13 artículos. La Tabla II resume los datos sobre AINES utilizados, su dosis, vía de administración, tiempo medido en minutos desde la administración del AINES hasta el inicio de la cirugía, duración de la intervención, analgésico de rescate utilizado y principal(es) conclusión(es).

\section{DISCUSIÓN}

En la presente revisión, se trató de establecer la eficacia y seguridad de la analgesia preoperatoria con AINES, en el control del dolor agudo postoperatorio, luego de desinclusiones quirúrgicas de terceros molares.

Savage \& Henry (2004), postulan que la manera más eficaz de comparar el efecto analgésico preoperatorio de un fármaco es evaluando el dolor postoperatorio experimentado, cuando el analgésico es administrado antes, y comparado con el mismo fármaco, administrado en la etapa postoperatoria. No todos los artículos que aquí son analizados cumplen con este requisito, y es por esta razón, que resulta difícil poder determinar la eficacia de la analgesia preoperatoria.
Por otro lado, existe también la dificultad que entrega la heterogeneidad de los medicamentos utilizados, si bien es cierto, el mecanismo de acción de todos los AINES es similar, es difícil establecer una conclusión comparándolos por igual, ya que tienen distintas eficacias y potencia; los artículos no las consideran y las dosis no son homogéneas. También hay que considerar que existen AINES selectivos y no selectivos.

En general, la vía de administración de los AINES en los artículos seleccionados fue oral, a excepción de Ong et al. (2004) y Çagıran et al. (2014), donde se usó la vía endovenosa, y en el estudio de Velásquez et al. (2014), se usó la vía intramuscular. La elección de la vía de administración de los AINES depende de algunos factores, como son: la rapidez del inicio analgésico y la necesidad con la que se requiere, tipo de analgésico, la capacidad de usar dosis tituladas y la biodisponibilidad, logrando con la vía parenteral intravenosa, un $100 \%$ de biodisponibilidad (Rosen \& Barkin, 1992). Teniendo en cuenta que los AINES son fármacos usados en dolores de intensidad leve a moderada, no se justificaría el uso de la vía parenteral en forma sistemática, ya que es más costoso, doloroso para el paciente, existe el riesgo de formación de hematomas y, por último, pero no menor, expone a los tratantes a potenciales accidentes cortopunzantes, por lo tanto, si la administración parenteral no tiene mayores beneficios que la oral, debería ser evitada (Schwartz et al., 2000).

Con respecto a la necesidad de un analgésico de rescate, se observó que el consumo total de analgésico fue menor, esto se explica porque la analgesia preoperatoria retrasa el inicio del dolor en la eta- 
pa postoperatoria, por lo tanto, la administración de AINES preoperatorios reduce el consumo de analgésicos de rescate en el postoperatorio. Esto nos sirve como indicador de éxito de la terapia analgésica preoperatoria.

En los estudios realizados por Aoki et al. (2006), Pektas et al. (2007) y Mojsa et al. (2017), se utilizaron fármacos del grupo oxicam (meloxicam, ampiroxicam y lornoxicam), los cuales poseen mayor inhibición sobre COX2. En adición, Chiu \& Cheung (2005) y Costa et al. (2015), utilizaron fármacos del grupo coxib (Rofecoxib y Etoricoxib respectivamente), siendo estos de un efecto más prolongado y analgésicos selectivos para COX2, lo que les da mayor capacidad para suprimir la hiperalgesia. Por otro lado, Sener et al. (2005) compararon el efecto de distintos AINES, dentro de los cuales incluye fármacos del grupo oxicam y coxib, no encontrando diferencias significativas entre ellos. El efecto preventivo encontrado por Chiu \& Cheung, y el menor consumo de analgésicos de rescate por parte del grupo pretratado con rofecoxib, se explicaría porque el rofecoxib tiene una duración prologada y el ibuprofeno es un analgésico de acción corta.

Ong et al., en su estudio, donde se administró en la etapa preoperatoria ketorolaco $30 \mathrm{mg}$ intravenoso, explicación que la disminución del dolor postoperatorio se debería a que el ketorolaco cubriría el peak del dolor producido en la etapa postoperatoria. Este fármaco tiene la peculiaridad de que se compara su potencia analgésica con $10 \mathrm{mg}$ de morfina, y se postula que además liberaría ligandos endogénos del receptor k-opiode (Flores, 2007).

Dentro de los estudios realizados por Ong et al., Chiu \& Cheung, Aoki et al., Çagiran et al. y Costa et al. se encontró que la analgesia preoperatoria, en comparación a un grupo control placebo, fue efectiva. Principalmente, sus resultados reflejaron un mayor tiempo en la aparición de dolor postoperatorio y una menor intensidad de este, viéndose reflejado también, en un menor consumo de analgésicos de rescate.

Por otro lado, Jung et al. (2005), Kaczmarzyc et al. (2010), Aznar-Arasa et al. (2012), Liporaci Junior (2012) y Mojsa et al., concluyen en sus estudios, que la analgesia preoperatoria no es efectiva (no se observó una diferencia significativa), comparándolos con un grupo que recibió analgesia postoperatoria. Esto se explicaría por la acción de enmascaramiento que ejercerían los anestésicos locales sobre el efecto anal- gésico de los AINES preoperatorio, coincidiendo algunas veces con el peak analgésico producido por el AINE, otra razón posible sería la rápida absorción del analgésico. Por esta razón, Jung et al. recomiendan seguir el patrón de administración de analgésicos de rescate en la etapa postoperatoria, siempre que éste sea administrado antes de que el dolor se desarrolle, $o$ antes de que finalice el efecto anestésico.

De los resultados obtenidos por Pektas et al., se puede inferir que la vida media del AINE influyó en el consumo de analgésicos de rescate por grupos, y por esta razón el grupo premedicado con lornoxicam $16 \mathrm{mg}$ oral, consumió mayor cantidad de analgésicos de rescate por ser de vida media corta, que el grupo premedicado con diflunisal $1000 \mathrm{mg}$ oral de vida media larga.

El ketoprofeno en un AINE ampliamente utilizado en cirugía oral. Velásquez et al. compararon el uso de ketoprofeno, versus el uso de diclofenaco, ambos vía intramuscular, y concluye que una dosis de 100 $\mathrm{mg}$ de ketoprofeno es más efectiva que $75 \mathrm{mg}$ de diclofenaco. Çagıran et al., por otro lado, evaluaron la efectividad del dexketoprofeno trometamol (DT), compuesto enantiómero del ketoprofeno, y determinó que el DT disminuye la intensidad del dolor y retrasa la necesidad de analgésicos de rescate.

Costa et al., además de evaluar el efecto analgésico del etoricoxib, fueron los únicos autores que evaluaron también la eficacia antiinflamatoria, mediante una categorización del edema con el uso de medidas faciales, en sus resultados no pudo obtener resultados significativos en la disminución del edema. Esto se puede explicar, ya que los AINES solamente disminuyen la formación de prostaglandinas, y en el sitio de la injuria tisular aparecen más mediadores de la inflamación, como quininas, histamina y bradiquinina, y para estos últimos los AINES no ejercen acción directa. Esta última afirmación, también puede explicar la ineficacia de la analgesia preoperatoria reportada por algunos autores.

CONCLUSIÓN. Si bien, varios autores seleccionados apoyan el uso de analgesia preoperatoria en desinclusión de terceros molares, no es posible determinar con certeza su eficacia, debido a la heterogeneidad de los AINES utilizados, las diferentes dosis, potencias y eficacias de estos. A esto debemos agregar, que existen fármacos como el rofecoxib, que ya no se encuentran en el mercado. Faltan estudios clí- 
nicos más homogéneos, que evalúen la analgesia preoperatoria frente a un grupo postoperatorio y un grupo placebo.

CARBALlOSA, F. U.; AGUILAR, Z. G.; PACHECO, M. C.1 \& FIGUEROA, C. L. Efficacy of preemptive analgesia with nonsteroidal anti-inflammatory drugs (NSAIDs) in third molar surgery. Literature review. Int. J. Odontostomat., 12(2):131-136, 2018.

SUMMARY: The management of postoperative pain in third molar surgery is fundamental. The use of nonsteroidal antiinflammatory drugs (NSAIDs) are considered an excellent alternative for pain management, administered both, postoperative and preoperative. The purpose of this review is to establish the efficacy and safety of preemptive analgesia with nonsteroidal antiinflammatory drugs in surgical disinclusions of third molars, in which at least partial bone was removed. We conducted a search in databases like: PubMed, EBSCO, Cochrane Library and Scielo; using the key words: Preemptive Analgesia, NSAID, Oral Surgery. Thirteen articles were selected, in total of 875 patients evaluated in the selected articles. Although several authors considered preemptive analgesia with NSAIDs as an effective method, more standardized studies are needed to demonstrate its effectiveness in oral surgery.

KEY WORDS: preemptive analgesia, nsaids, oral surgery.

\section{REFERENCIAS BIBLIOGRÁFICAS}

Aoki, T.; Yamaguchi, H.; Naito, H.; Shiiki, K.; Izawa, K.; Ota, Y.; Sakamoto, H. \& Kaneko, A. Premedication with cyclooxygenase-2 inhibitor meloxicam reduced postoperative pain in patients after oral surgery. Int. J. Oral Maxillofac. Surg., 35(7):613-7, 2006.

Aznar-Arasa, L.; Harutunian, K.; Figueiredo, R.; Valmaseda-Castellón, E. \& Gay-Escoda, C. Effect of preoperative ibuprofen on pain and swelling after lower third molar removal: a randomized controlled trial. Int. J. Oral Maxillofac. Surg., 41(8):1005-9, 2012.

Çagıran, E.; Eyigör, C.; Sezer, B. \& Uyar, M. Preemptive analgesic efficacy of dexketoprofen trometamol on impacted third molar surgery. Agri, 26(1):29-33, 2014.

Campbell, W. I.; Kendrick, R. \& Patterson, C. Intravenous diclofenac sodium. Does its administration before operation suppress postoperative pain? Anaesthesia, 45(9):763-6, 1990.

Chiu, W. K. \& Cheung, L. K. Efficacy of preoperative oral rofecoxib in pain control for third molar surgery. Oral Surg. Oral Med. Oral Pathol. Oral Radiol. Endod., 99(6):e47-53, 2005.

Costa, F. W.; Soares, E. C.; Esses, D. F.; Silva, P. G.; Bezerra, T. P.; Scarparo, H. C.; Ribeiro, T. R. \& Fonteles, C. S. A split-mouth, randomized, triple-blind, placebo-controlled study to analyze the preemptive effect of etoricoxib $120 \mathrm{mg}$ on inflammatory events following removal of unerupted mandibular third molars. Int. J. Oral Maxillofac. Surg., 44(9):1166-74, 2015.

Flores, J. Tratamiento Farmacológico del Dolor. Barcelona, Ars Médica, 2007.

Grape, S. \& Tramèr, M. R. Do we need preemptive analgesia for the treatment of postoperative pain? Best Pract. Res. Clin. Anaesthesiol., 21(1):51-63, 2007.
Jung, Y. S.; Kim, M. K.; Um, Y. J.; Park, H. S.; Lee, E. W. \& Kang, J. W. The effects on postoperative oral surgery pain by varying NSAID administration times: comparison on effect of preemptive analgesia Oral Surg. Oral Med. Oral Pathol. Oral Radiol. Endod., 100(5):559-63, 2005.

Kaczmarzyk, T.; Wichlinski, J.; Stypulkowska, J.; Zaleska, M. \& Woron, J. Preemptive effect of ketoprofen on postoperative pain following third molar surgery. A prospective, randomized, double-blinded clinical trial. Int. J. Oral Maxillofac. Surg., 39(7):647-52, 2010.

Katz, J. George Washington Crile, anoci-association, and pre-emptive analgesia. Pain, 53(3):243-5, 1993.

Kelly, D. J.; Ahmad, M. \& Brull, S. J. Preemptive analgesia I: physiological pathways and pharmacological modalities. Can. J. Anaesth., 48(10):1000-10, 2001a.

Kelly, D. J.; Ahmad, M. \& Brull, S. J. Preemptive analgesia II: recent advances and current trends. Can. J. Anaesth., 48(11):1091-101, 2001b.

Kissin, I. Preemptive analgesia. Anesthesiology, 93(4):1138-43, 2000.

Liporaci Junior, J. L. Assessment of preemptive analgesia efficacy in surgical extraction of third molars. Rev. Bras. Anestesiol., 62(4):502-10, 2012.

Mojsa, I. M.; Stypulkowska, J.; Novak, P.; Lipczynski, K.; Szczeklik, K. \& Zaleska, M. Pre-emptive analgesic effect of lornoxicam in mandibular third molar surgery: a prospective, randomized, double-blind clinical trial. Int. J. Oral Maxillofac. Surg., 46(5):614-20, 2017.

Ong, K. S.; Seymour, R. A.; Chen, F. G. \& Ho, V. C. Preoperative ketorolac has a preemptive effect for postoperative third molar surgical pain. Int J. Oral Maxillofac. Surg., 33(8):771-6, 2004.

Pektas, Z. O.; Sener, M.; Bayram, B.; Eroglu, T.; Bozdogan, N.; Donmez, A.; Arslan, G. \& Uckan, S. A comparison of pre-emptive analgesic efficacy of diflunisal and lornoxicam for postoperative pain management: a prospective, randomized, single-blind, crossover study. Int. J. Oral Maxillofac. Surg., 36(2):123-7, 2007.

Romero-Ruiz, M. M.; Herrero-Climent, M.; Torres-Lagares, D. \& GutiérrezPérez, J. L. Protocolo de control del dolor y la inflamación postquirúrgica. Una aproximación racional. R. C. O. E., 11(2):205-15, 2006.

Rosen, P. \& Barkin, R. M. Emergency Medicine. $3^{\text {rd }}$ ed. St. Louis, Mosby, 1992. pp.201-28.

Savage, M. G. \& Henry, M. A. Preoperative nonsteroidal anti-inflammatory agents: review of the literature. Oral Surg. Oral Med. Oral Pathol. Oral Radiol. Endod., 98(2):146-52, 2004.

Schwartz, N. A; Turturro, M. A.; Istvan, D. J. \& Larkin, G. L. Patients perceptions of route of nonsteroidal anti-inflammatory drug administration and its effect on analgesia. Acad. Emerg. Med., 7(8):85761, 2000.

Sener, M.; Ozgur Pektas, Z.; Yilmaz, I.; Turkoz, A.; Uckan, S.; Donmez, A. \& Arslan, G. Comparison of preemptive analgesic effects of a single dose of nonopioid analgesics for pain management after ambulatory surgery: A prospective, randomized, single-blind studyin Turkish patients. Curr. Ther. Res. Clin. Exp., 66(6):541-51, 2005

Velásquez, G. C.; Santa Cruz, L. A. \& Espinoza, M. A. Ketoprofen is more effective than diclofenac after oral surgery when used as a preemptive analgesic: a pilot study. J. Oral Facial Pain Headache, 28(2):153-8, 2014.

Woolf, C. J. \& Chong, M. S. Preemptive analgesia--treating postoperative pain by preventing the establishment of central sensitization. Anesth. Analg., 77(2):362-79, 1993

\section{Dirección para correspondencia}

Dr. Gerardo Ignacio Aguilar Zavala

Cirujano Dentista

Universidad de los Andes

Santiago - CHILE

Email:giaguilarz@gmail.com

Recibido : 04-12-2017

Aceptado: 07-02-2018 